\title{
Unfavorable Hodgkin Lymphoma
}

National Cancer Institute

\section{Source}

National Cancer Institute. Unfavorable Hodgkin Lymphoma. NCI Thesaurus. Code C68663.

A Hodgkin lymphoma with unfavorable prognosis. 